\title{
Analysis on Benefit Risk Factors of Creative Industrial Park Based on Fuzzy Comprehensive Evaluation Yong $\mathrm{Li}^{1,2}$, Qian $\mathrm{Wu}^{1,2}$, Xin $\mathrm{Hu}^{1,2}$
}

${ }^{1}$ School of Civil Engineering, Xi`an University of Architecture and Technology, Xi`an 710055, China

${ }^{2}$ Department of Architecture and Civil Engineering, Xi`an University of Architecture and Technology, Xi`an 710055, China

\section{基于模糊综合评判的创意产业园 效益风险因素分析

\author{
李雍 ${ }^{1,2}$, 武乾 ${ }^{1,2}$, 胡变 ${ }^{1,2}$ \\ 1 西安建筑科技大学土木工程学院, 西安, 710055, 中国 \\ 2 西安建筑科技大学建筑与土木工程系, 西安, 710055, 中国
}

\begin{abstract}
The transformation and reuse of old industrial buildings as creative industry parks has become the mainstream trend. In order to prevent more companies from following blindly, this article starts from the perspective of the company and conducts an on-site inspection of the Hua Qing Science and Education Industrial Park in Shaanxi Province. The fuzzy comprehensive evaluation method is used to analyze the profitability of its operation stage, weights are determined by combination weighting method, and the risk factors that affect its effectiveness are analyzed to provide reference for the future development of creative industrial parks. The paper identifies the risk factors affecting economic efficiency mainly as its project management model; the main risk factors affecting social benefits are its ability to enhance regional image; the main risk factors affecting environmental benefits are the
\end{abstract}

performance of green building operations management. Enterprises should judge their own capabilities based on relevant risk factors, and then more rationally choose the mode of space utilization for building renovation.

Keywords: Old industrial building; Fuzzy Comprehensive Evaluation; Creative Industrial Park; Combined empowerment method; Benefit risk factors

摘要

现今旧工业建筑改造再利用为创意产 业园已成为主流趋势。为避免更多企业盲目 追随, 本文从企业的视角出发, 对陕西省华 清科教集团实地考察。运用模糊综合评判法 分析其运营阶段效益情况, 采用组合赋权法 确定权重, 分析影响其效益的风险因素, 为 今后创意产业园的发展提供参考性意见。文 中确定出影响经济效益的风险因素主要是 其项目管理模式; 影响社会效益的主要风险 因素是对其区域形象提升能力; 影响环境效 益的主要风险因素是绿色建筑运营管理表 现能力。企业应根据相关风险因素判断自身 
能力, 进而更合理地选择建筑改造空间利用 模式。

关键词：旧工业改造; 模糊综合评判; 创意 产业园; 组合赋权; 效益风险因素

\section{1. 引言}

在中国新政策的催生下, 文化创意产业 园的发展已成为传统经济转型的一个重要 引导方向。一方面, 文化创意产业园可以有 效的保存一个国家一个城市一代人的生存 印记, 另一方面, 文化创意产业园与现代元 素的融合给新技术带去新的灵感, 提升区域 竞争力。但在文化创意产业园发展的过程中 也存在着许多问题, 比如其经济效益、社会 效益和环境效益是否达到预期的效果; 许多 地方为规避风险, 盲目跟风, 进行文化创意 产业园的改造是否正确; 文化创意产业园本 身代表的文化的局限性能否吸引其他行业 的注册等。本文将以陕西省华清科教产业园 为例对文化创意产业园的改造所带来的经 济、社会、环境效益进行分析, 取得各效益 的影响因素, 为文化创意产业园的发展提供 建议, 为入驻公司的经营管理提供参考。陕 西省华清科教产业园是中国西北最具示范 性的设计创意主题产业园, 对其的效益风险 因素分析具有很好的借鉴意义。目前对其效 益评价的方法有很多, 比如专家评分法、德 尔菲法、TOPSIS 法、人工神经网络法、可 拓优度评价等 ${ }^{[1]}$, 本文将采用模糊综合评价 法 $^{[5]}$, 利用组合赋权的方法确定权重, 建立 关于文化创意产业园的效益风险因素模型, 并为创意产业园的发展提出相关性建议。

\section{2. 文化创意产业园的效益风险因素构成}

\section{1 风险因素构建原则及基本框架}

对文化创意产业园效益风险因素的研 究是衡量其项目经实践活动所达到预期目 标和指标实现的情况, 从其效益评价的的阶 段来说, 属于项目后评价的范畴, 其主要作 用在于判断从策划、实施到运营整个项目的 经济、社会和环境等效益是否达到预期目 标, 从发现的问题中获取经验知识, 为后期 项目决策提供有效、可靠的参考信息, 提高 决策水平 ${ }^{[1][2]}$ 。构建其风险因素需要满足系
统性、科学性、规范性、客观性及全面性等 原则 ${ }^{[7]}$, 本文参考文献并结合对部分创意产 业园的实际调研确立了如下风险因素：风险 因素由目标层、项目层和标准层 3 个层次构 成。目标层为综合风险因素——创意产业园 效益风险因素; 项目层包括 3 个子目标,即经 济效益、社会效益、环境效益;标准层共有 18 项指标,全面反映影响文化创意产业园的 各个方面 ${ }^{[7]}$ 。具体见表 1 。

表 1 创意产业园效益风险因素

\begin{tabular}{|c|c|c|}
\hline $\begin{array}{c}\text { 目标层 } \\
\text { X }\end{array}$ & 项目层 $\mathrm{U}_{\mathrm{i}}$ & 标准层 $\mathrm{U}_{\mathrm{ij}}$ \\
\hline $\begin{array}{c}\text { 创意产 } \\
\text { 业园效 } \\
\text { 益风险 } \\
\text { 因素 }\end{array}$ & $\begin{array}{c}\text { 社会效益 } \\
U_{2}\end{array}$ & $\begin{array}{c}\text { 工程检测成本 } U_{11} \\
\text { 建筑结构加固成本 } U_{12} \\
\text { 项目投资计划 } U_{13} \\
\text { 项目融资模式 } U_{14} \\
\text { 项目管理模式 } U_{15} \\
\text { 物业成本 } U_{16} \\
\text { 园区主题定位 } U_{21} \\
\text { 与周围环境协调性 } U_{22} \\
\text { 提供就业机会能力 } U_{23} \\
\text { 对区域形象提升能力 } \\
\text { U } \\
\text { 对区域经济发展影响 } \\
\text { } U_{25} \\
\text { 对自然、历史、文化遗 } \\
\text { 产保护程度 } U_{26} \\
\text { 与区域地理环境的结合 } \\
\text { 程度 } U_{31} \\
\text { 对可再生能源的利用程 } \\
\text { 度 } U_{32} \\
\text { 噪音污染程度 } U_{33} \\
\text { 绿色建筑运营管理表现 } \\
\text { 低程度 } U_{36} \\
\text { 新材料的运用程度 } U_{35} \\
\text { 节能措施对总能的降 }\end{array}$ \\
\hline
\end{tabular}

\section{2 风险因素说明}

\section{2 .1 经济效益}

该一级风险因素细分为 6 各层级: (1)工 程检测成本: 由于旧工业建筑自身的特性, 建筑在确定能否再利用前均需对建筑结构 检测, 这部分成本是项目改造总成本中的首 要组成部分。(2)建筑结构加固成本: 检测完 成后的建筑物存在各种问题, 在必须利用的 建筑结构部分不达标的情况下必然花费的 成本。(3)项目投资计划: 影响项目过程中投 资计划的合理性。(4)项目融资模式: 项目投 资模式对工程项目影响非常大, 与项目及投 
资方性质的贴合程度对项目经济效益的影 响程度很大。(5)项目管理模式: 管理贯穿于 项目的各个阶段, 对项目的收益影响重大。 (6)物业成本: 项目在运营过程中物业费占据 了很大比例, 对经济效益产生很大的影响。 2.2.2 社会效益

(1)园区主题定位：园区的主题定位直接 影响到入驻企业的类型, 是带动区域定位的 一个重要组成部分。(2)与周围环境协调性: 主要考虑项目运营后与周围社会环境的协 调匹配程度。(3)提供就业能力: 反映创意产 业园的行业吸引力及对就业情况的贡献度。 (4)对区域形象的提升能力: 一个园区可以成 为一个区域发展的领头羊, 通过此影响因素 可以反映区域形象的贡献程度。(5)对区域经 济发展影响: 反映产业园对区域居民收入及 生活水平提高的显著程度。(6)对自然、历史、 文化遗产的保护程度: 旧工业创意产业园是 对历史印记的有效保留, 对自然、历史、文 化遗产的保护程度直接影响它的社会价值。 2.2.3 环境效益

(1)与区域地理环境的结合程度: 考虑因 地制宜的原则, 通过与区域地理环境的结合 程度反映项目利用地理优势的情况。(2)对可 再生能源的利用程度: 反映建筑物对太阳 能、风能等的利用能力。(3)噪音污染程度: 项目在改造过程中会产生很大程度的噪音 污染, 运营维护阶段也仍然会进行小范围的 装饰装修, 以适应园区的发展需要, 期间产 生的噪音污染也不容忽视。(4)绿色建筑运营 管理表现: 在建筑运营管理阶段的管理方法 及制度等的合理及有效性的分析能很好的 反映环境效益的好坏程度。(5)新材料的运用 程度：旧工业建筑改造时对新材料的运用对 环境的影响尤其室内环境影响很大。(6)节能 措施对总能耗的降低程度: 节能措施对总能 耗的降低程度是影响环境效益的不可或缺 的因素。

\section{3. 构建基于模糊综合评价法的数学模型}

模糊综合评判法是运用模糊数学的模 糊变换基本原理和累计隶属度原则, 是多重 目标综合评判决策的一种特殊情形, 它考虑 与被评价事物相关的各种因素, 对方案进行 综合评价 ${ }^{[8][4]}$ 。该法采用方案的各项定量指
标及定性指标, 集中专家和评价者的经验及 智慧, 对方案进行综合分析评价 ${ }^{[8]}$ 。本文在 确定各项指标权重时采用主观与客观相结 合的方法, 使得结果更加具有客观性和准确 性 ${ }^{[8]}$ 。对具体事物建立评价因素集、各因素 权重集、各种评价结果组成的评价集,采用一 定的合成算子进行综合运算和评价. 其基本 步骤如下:

1)确定评价因素集 $\mathrm{U}=\left\{\mathrm{u}_{1}, \mathrm{u}_{2}, \cdots, \mathrm{u}_{\mathrm{n}}\right\}$, 其中 $\mathrm{u}_{\mathrm{i}}(\mathrm{i}=1,2, \cdots, \mathrm{n})$ 表示影响事物评价值的第 $\mathrm{i}$ 个 因素.

2)确定评价等级集 $\mathrm{V}=\left\{\mathrm{v}_{1}, \mathrm{v}_{2}, \cdots, \mathrm{v}_{\mathrm{m}}\right\}$, 其 中 $\mathrm{v}_{\mathrm{j}}(\mathrm{j}=1,2, \cdots, \mathrm{s})$ 表示影响事物评价值的第 $\mathrm{j}$ 个等级.

3)确定指标隶属度, 构造隶属矩阵 R. 指 标值越大, 影响主体的程度越高, 此时采用 升半梯形分布函数来确定隶属度, 否则采用 降半梯形分布函数, 进而形成隶属矩阵 ${ }^{[9]}$ 。

4)确定各因素的权重. 本文采用 AHP 法 与熵权法相结合的方式确定各指标的权重:

(1)确定熵权 $\mathrm{W}^{(1)}=\left(\mathrm{a}_{1}, \mathrm{a}_{2}, \cdots, \mathrm{a}_{\mathrm{n}}\right)$. 假定有 $\mathrm{m}$ 个待评价的样本, 每个待评价样本有 $\mathrm{n}$ 个评 价指标, 则有样本的判断矩阵 $\mathrm{R}=\left(\mathrm{x}_{\mathrm{ij}}\right) \mathrm{n} \times \mathrm{m}$ $(i=1,2, \cdots, n ; j=1,2, \cdots, m)$. 式中, $x_{i j}$ 为经过的归一 化处理后第 $\mathrm{i}$ 个样本第 $\mathrm{j}$ 个指标值, 根据熵的 定义, 确定各评价指标的摘为 ${ }^{[11]}$

$$
\begin{aligned}
& H_{j}=-\left(\sum_{i=1}^{m} f_{i j} \ln f_{i j}\right) / \ln m \\
& i=1,2,3, \ldots, m ; j=1,2, \ldots, n \\
& \text { 式中 } f_{i j}=x_{i j} / \sum_{i=1}^{m} x_{i j} \\
& \text { 且规定当 } f_{i j}=0 \text { 时, } f_{i j} \ln f_{i j}=0, \text { 相应的 }
\end{aligned}
$$

第 $j$ 个指标的熵权定义为

$a_{j}=\left(1-H_{j}\right) /\left(n-\sum_{j=1}^{n} H_{j}\right), 0 \leq a_{j} \leq 1$, 且 $\sum_{j=1}^{n} a_{j}=1$.

(2)AHP 法确定权重 $\mathrm{W}^{(2)}$ 。首先建立层次 结构模型。将目标层、项目层、准则层分为 最高层、中间层和最底层, 绘制层次结构模 型; 其次构造判断矩阵。邀请专家依据经验 独立地对各项指标的相对重要程度进行打 分; 再根据判断矩阵计算权重。权重的计算 方法是求出判断矩阵最大特征值对应的特 征向量, 然后将其特征向量归一化; 层次单 排序并对其一次性检验。由判断矩阵计算同 
一层次的各因素相对于上一层中某一因素 的重要性权重, 进行层次单排序, 并对层次 单排序做一致性检。一致性指标为: $C I=\frac{\lambda_{\text {max }}-n}{n-1}$, 平均随机一次性指标 RI 则根 据表格所得, 当 $\mathrm{CR}=\mathrm{RI} / \mathrm{CI}<0.1$ 时, 满足一 致性检验。

(3)组合权重 W。在此使用拉格朗日乘 子法优化可得组合权重计算式 ${ }^{[10]}$ :

$$
W=\frac{\left(W^{(1)} W^{(2)}\right)^{1 / 2}}{\sum_{i=1}^{\mathrm{s}}\left(W^{(1)} W^{(2)}\right)^{1 / 2}} i=1,2, \ldots, \mathrm{S}
$$

5)综合评价. 将各指标的权重和隶属度矩 阵带入 $B=W \delta R=\left(b_{1}, b_{2}, \cdots, b_{s}\right)$ 其中 $\delta$ 为模 糊合成算子, 表示模糊矩阵的合成运算,采用 加权平均型模糊合成算子。按最大隶属度原 则, 选取 B 中最大的隶属度所对应的评语作 为该方案的总评价。

\section{4. 实例应用}

陕西省华清科教文化创意产业园发展 有限公司为了有效保护和利用原陕钢遗留 工业建筑群, 对原陕钢特钢车间老厂房重新 定位、规划、改造。现园区总占地面积 50 亩, 改造后总建筑面积约 4.5 万平方米, 单 元面积 100 平方米一 2000 平方米, 层高达 3.9 米一7.8 米, 绿化覆盖率 30\%。在 2012 年, 华清集团将现有老钢厂园区定位为文化 创意产业园, 主要入驻企业类型有设计类、 文创类、互联网科技等创新类产业。它具有 重新界定城市文脉、对周边区域后期改造定 位与借鉴的作用, 是集市民休闲与创意产业 为一体的城市新坐标。对其利用相应影响因 素的分析来确定其自改造至目前的效益状 况 ${ }^{[7]}$ 。

1) 确定因素集和评价集。根据影响因 素列表共 18 项, 故 $n=18$ 。令评价集 $V=\left\{\mathrm{v}_{1}, \mathrm{v}_{2}\right.$, $\left.\mathrm{v}_{3}, \mathrm{v}_{4}, \mathrm{v}_{5}\right\}$, 其中 $\mathrm{v}_{1}$ 代表优秀、 $\mathrm{v}_{2}$ 代表良好、 $\mathrm{v}_{3}$ 代表中等、 $\mathrm{v}_{4}$ 代表较差、 $\mathrm{v}_{5}$ 代表很差。

2) 样本归一化处理。邀请了 15 位专家 对创意产业园的效益影响因素的重要程度 进行打分, 得到的原始数据, 经

$$
\mathrm{x} i j=\frac{x_{i j}^{*}-\min x_{i j}^{*}}{\max x_{i j}^{*}-\min x_{i j}^{*}} \text { (越大越优型), }
$$

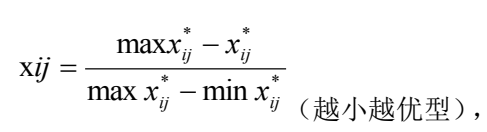
将其归一化得 $\mathrm{x}_{\mathrm{ij}}{ }^{[11]}$ 。

3) 确定评判隶属矩阵 $R$ 。利用已经归一 化的评价指标, 取与之相对应的评级等级标 准 $[0.8,1] 、[0.6,0.8) 、[0.4,0.6) 、[0.3,0.4)$ 、 $[0.2,0.3)$ 。建立评判矩阵 $R=(R 1, R 2, R 3) T$, 其 中

$$
R_{1}=\left[\begin{array}{ccccc}
0.562 & 0.438 & 0 & 0 & 0 \\
0.701 & 0.299 & 0 & 0 & 0 \\
0.312 & 0.679 & 0.009 & 0 & 0 \\
0.288 & 0.7 & 0.012 & 0 & 0 \\
0.363 & 0.551 & 0.086 & 0 & 0 \\
0.436 & 0.52 & 0.044 & 0 & 0
\end{array}\right]
$$$$
R_{2}=\left[\begin{array}{ccccc}
0.502 & 0.497 & 0.001 & 0 & 0 \\
0.287 & 0.654 & 0.059 & 0 & 0 \\
0.377 & 0.55 & 0.073 & 0 & 0 \\
0.3 & 0.697 & 0.003 & 0 & 0 \\
0.203 & 0.598 & 0.199 & 0 & 0 \\
0.329 & 0.646 & 0.025 & 0 & 0
\end{array}\right]
$$$$
R_{3}=\left[\begin{array}{ccccc}
0.238 & 0.762 & 0 & 0 & 0 \\
0.6 & 0.335 & 0.065 & 0 & 0 \\
0.75 & 0.25 & 0 & 0 & 0 \\
0.407 & 0.51 & 0.083 & 0 & 0 \\
0.105 & 0.615 & 0.28 & 0 & 0 \\
0.331 & 0.619 & 0.05 & 0 & 0
\end{array}\right]
$$

4) 确定各影响因素权重。根据熵权法 确定的权重为 $\mathrm{W}^{(1)}$, AHP 法计算所得的权重 为 $\mathrm{W}^{(2)}$, 并通过拉格朗日法确定了其组合权 重。具体数值如表 2 。

5) 综合评价。将标准层权重和隶属度 矩阵代入 $U=H \delta R$, 得项目层模糊评级为

再将项目层权重代入 $B=G \delta C$, 得模糊综 合评价结果为 $\mathrm{B}=(0.3455,0.6116,0.0429,0,0)$

根据定义的评定等级和最大隶属度的 原则, 可以得出陕钢厂创意产业园的总体效 益处于良好水平; 项目层中, 经济效益、社 会效益均为良好状态, 有待加强; 环境效益 处于中等水平, 有待改进完善。总体而言, 陕钢厂创意产业园效益状况与实际水平相 
表 2 影响因素权重计算结果

\begin{tabular}{|c|c|c|c|c|c|c|}
\hline 项目层 X & 权重 G & 标准层 Ui & AHP 法权重 $\mathrm{W}^{(2)}$ & 熵权 W $\mathrm{W}^{(1)}$ & 标准层权重 H & 组合权重 W \\
\hline \multirow{6}{*}{$\mathrm{U}_{1}$} & \multirow{6}{*}{0.4047} & U11 & 0.0217 & 0.0043 & 0.0374 & 0.0104 \\
\hline & & U12 & 0.0131 & 0.0341 & 0.0348 & 0.0227 \\
\hline & & U13 & 0.0685 & 0.0138 & 0.1494 & 0.0329 \\
\hline & & U14 & 0.0665 & 0.0177 & 0.1093 & 0.0367 \\
\hline & & U15 & 0.1833 & 0.3195 & 0.4262 & 0.2594 \\
\hline & & U16 & 0.1029 & 0.1044 & 0.2429 & 0.1111 \\
\hline \multirow{6}{*}{$\mathrm{U}_{2}$} & \multirow{6}{*}{0.3145} & $\mathrm{U} 21$ & 0.0279 & 0.0783 & 0.0766 & 0.0501 \\
\hline & & $\mathrm{U} 22$ & 0.0149 & 0.0119 & 0.1092 & 0.0142 \\
\hline & & $\mathrm{U} 23$ & 0.0388 & 0.0189 & 0.2295 & 0.0290 \\
\hline & & U24 & 0.0751 & 0.1219 & 0.2414 & 0.1026 \\
\hline & & U25 & 0.0667 & 0.0891 & 0.1461 & 0.0826 \\
\hline & & U26 & 0.0321 & 0.0462 & 0.1972 & 0.0413 \\
\hline \multirow{6}{*}{$\mathrm{U}_{3}$} & \multirow{6}{*}{0.2808} & U31 & 0.0491 & 0.0101 & 0.0189 & 0.0239 \\
\hline & & U32 & 0.0663 & 0.0498 & 0.1890 & 0.0616 \\
\hline & & U33 & 0.0121 & 0.0011 & 0.0978 & 0.0039 \\
\hline & & U34 & 0.1001 & 0.0610 & 0.3836 & 0.0837 \\
\hline & & U35 & 0.0200 & 0.0020 & 0.1129 & 0.0068 \\
\hline & & U36 & 0.0406 & 0.0159 & 0.0978 & 0.0272 \\
\hline
\end{tabular}

符, 评价结果具有一定的可信度。

$$
U=\left\lfloor\begin{array}{l}
U_{1} \\
U_{2} \\
U_{3}
\end{array}\right\rfloor=\left\lfloor\begin{array}{lllll}
0.3628 & 0.6162 & 0.0209 & 0 & 0 \\
0.3233 & 0.6187 & 0.0580 & 0 & 0 \\
0.3454 & 0.5971 & 0.0575 & 0 & 0
\end{array}\right\rfloor
$$

\section{5. 结论及建议}

从子系统评价结果和综合评价结果来看, 陕西省华清科教集团总体上是比较成功的, 但在 “环境效益”方面表现一般。

(1) 通过分析, 我们可以看到经济效益 的风险因素主要是项目管理模式和物业成本, 企业在运营期间的成本是衡量其效益成果的 关键阶段, 企业需要组成更加精锐的队伍来严 格制定相关管理制度, 及时调整项目管理方 案, 综合多方面因素管理创意产业园。工程检 测费用和建筑物结构加固费用在改造总成本 中虽占费用不大, 但却应该成为旧工业建筑改 造为创意产业园过程中一个长期的过程, 运营 期间做好定期检查维护。目前陕西文化创意产 业处于发展初级阶段, 未来发展中应该注重对 整体产业链价值的提升、开展网络化发展模 式, 纵向加深产业开发程度、横向扩展产业的 包容弹性 ${ }^{[11]}$, 培养专业人才、推广陕西省的特 色文化、增加产业对经济发展的提升作用。

(2) 其影响社会效益的主要风险因素是
对区域形象的提升能力、提供就业机会能力和 对区域经济发展影响能力。创意产业园存在的 目的之一就是能够为政府和社会的所做出的 贡献度, 除了经济的影响外, 解决青年创业就 业这项问题也是重中之重。目前已经有多个创 意产业园成为了区域地标性建筑, 企业在发展 自身的同时还应积极与周围环境相协调, 引领 城市发展趋势。除此之外, 文化创意产业园的 社会效益评价还应兼顾社会责任的承担、社会 支持度、社会贡献度、示范效应以及文化设施 等的覆盖率 ${ }^{[12]}$ 。从企业的角度出发, 企业应及 时根据政府政策导向, 及时调整企业定位, 寻 求特色文化, 将老一辈的思想或者技艺巧妙地 融合在改造当中, 那些应该保留的和必须完全 保留的记忆绝对不能舍弃, 牢牢抓住老一辈人 怀念的心、新生代的好奇心, 集人心再至地利 天时。

（3）影响环境效益的主要风险因素是对 可再生能源的利用程度和绿色建筑运营管理 表现。在环境质量日趋下滑的大背景下, 可再 生能源扮演着非常重要的角色, 太阳能、风能、 地热能、潮汐能等不断地再被挖掘、使用, 但 对于创意产业园来说使用效率还是处于较低 水平, 尤其因旧工业建筑物的历史较为悠久, 墙体、梁柱等结构有磨损, 想要在保存原有建 筑风味的基础上, 利用新型材料和新的施工技 
术将成为一大阻碍, 且电力不能够维持正常运 转, 对其线路的整改大多仍按普通方式, 无法 有效利用新能源。旧工业建筑尤其是老厂房, 其外围护结构一般不具有良好的保温隔热效 果, 在改造后, 其供热供暖等将耗费大量的能 源, 企业若要在此方面做到节约资源, 就必须 站在长远地角度, 制定完善合理的措施, 邀请 专业人士做相关改造, 以便在达到良好效果的 同时能够有效得节约资金。

综合以上三点, 创意产业园尤其是由旧工 业建筑改造的创意产业园无论是在建造还是 运营方面都有着较大的难题, 而随着改造项目 的逐渐增多, 相关技术的不断完善, 其风险在 发展潮流中不断被削弱, 但要注意的是, 并不 是所有的旧工业建筑改造为创意产业园都是 合理的, 别具特色的、适合自身的改造模式才 是企业应该全力追求的。企业以长远的目光看 项目、准确识别改造项目背景、准确定位并结 合政策引导、顺从人心才能在经济、社会、环 境效益方面达到良好的效果, 且与其他发展项 目形成整体, 形成规模, 打造特色改造项目群 才是企业应该追求的目标。

\section{Acknowledgements}

This study was supported by National Natural Science Foundation of China (No. 51678479).

\section{致谢}

本研究得到了国家自然科学基金项目 (51678479)的资助。

\section{参考文献}

[1] 武乾,王冲,郑德志,马宏飞.基于主成分分 析的西安旧工业建筑再利用风险因素研 究.工业建筑,2014,44(10):61-63+90.

[2] 余晓松.旧工业建筑改造再利用全过程风 险管理研究.西安建筑科技大学, 2015 .

[3] 徐汉明,周筬. 基于环境效度影响因素分析 下的创意产业园区评估指标体系研究.中 国软科学, 2017,(03):164-177.

[4] 田冬梅,白丽华.基于模糊 AHP 模型的都 市创意产业园适应性评价研究. 企业导 报,2010(1) : 291-293.

[5] Wen F F, Li L. Based on AHP- fuzzy comprehensive evaluation method of real estate investment risk research.
Proceedings of the 2nd International Conference on Systems Engineering and Modeling (ICSEM-13), 2013,0413-0417.

[6] 李慧民,田卫,间瑞琦.旧工业建筑(群)再生 利用评价指标体系构建. 西安建筑科技大 学学报(自然科学版),2013, 45 (06): 772777.

[7] 杨玥.西安老钢厂创意产业园内旧工业建 筑空间再利用现状分析与研究. 建筑与文 化,2017,(09):89-90.

[8] 徐斌,黄显峰.基于模糊综合评判法的水利 现代化评价指标体系研究.水利发展研 究,2016,16(10):22-29+47.

[9] 刘大海,宫伟等. 基于 AHP-熵权法的海岛 海岸带脆弱性评价指标权重综合确定方 法.海洋环境科学,2015,34(03): 462-467.

[10] 高玉琴,陆晓华,黄祚继. 基于模糊综合评 价法的水库管理现代化评价及其应用.三 峡大学学报 (自然科学版),2017,39 (06):35-39.

[11] 李淑杰. 基于熵权模糊综合评价法的城中 村改造项目风险管理研究. 华北水利水电 大学,2016.

[12] 王怀,奕峰.上海中心城区市属创意产业 园区的发展绩效研究.上海城市规 划,2013,(1). 